\title{
Can Carpobrotus edulis, heal incisional and excisional wounds on the skin?
}

\author{
Gunal MY'1,7* Ayla $\mathrm{S}^{2,7^{*}}$, Caglayan $\mathrm{B}^{3,7}$, Beker $\mathrm{MC}^{3,7}$, Bedri $\mathrm{N}^{2}$, Aslan $\mathrm{I}^{4}$, Ozdemir $\mathrm{EM}^{5}$, \\ Kilic $E^{3,7}$, Yesilada $E^{6}$
}

Alanya Alaaddin Keykubat University, School of Medicine, Department of Physiology,

Antalya, Turkey.drmygunal@gmail.com

\begin{abstract}
OBJECTIVES: This study aimed to investigate the wound healing activity of liposomal Carpobrotus edulis powder extract (CEPE) formulation on incisional and excisional wounds in rat.

BACKGROUND: In the event of any damage, the damaged tissue undergoes a process of regenerating itself, which is called wound healing.

METHODS: Centella asiatica extract (CAE) was used as the reference molecule in the study. The wound healing process was tested by using the excisional and incisional wound model. On the 12th day of the study, maximum stress, stress, \% of elongation values were evaluated in the incisional wound. Also; histological parameters and macroscopic photographic analyses were evaluated in the excisional wound.

RESULTS: In the photo evaluations, the improvement was more prominent in both CAE and CEPE groups than in the control group. Histological evaluation showed that CEPE group had significant wound healing activity compared to the control and CAE groups. Axial tensile-elongation experiments in incisional wound tissue show that there was no significant difference between CAE and CEPE groups.

CONCLUSION: Liposomal formulations of C.edulis extract were found to have positive effects on the healing process, both on excisional and incisional wound tissues (Tab. 2, Fig. 3, Ref. 30). Text in PDF www.elis.sk KEY WORDS: Carpobrotus edulis, liposome, incisional wound, excisional wound, axial tensile-elongation tests.
\end{abstract}

\section{Introduction}

In the event of any damage, the damaged tissue undergoes a process of regenerating itself, which is called wound healing. There are hemostasis, inflammation, granulation tissue formation, neovascularization, reepithelization, and remodeling stages in the wound healing process (1). There are different experimental models to evaluate the wound healing process. Excisional and incisional wound models are commonly used in the literature. Surface area healing rates in excisional wound models; stress, strain, and percent elongation levels are evaluated by measuring wound tension

${ }^{1}$ Alanya Alaaddin Keykubat University, School of Medicine, Department of Physiology, Antalya, Turkey, ${ }^{2}$ Istanbul Medipol University, School of Medicine, Department of Histology-Embryology, Istanbul,Turkey, ${ }^{3}$ Istanbul Medipol University, School of Medicine, Department of Physiology, Istanbul, Turkey, ${ }^{4}$ University of Health Sciences, Department of Pharmacy, Istanbul,Turkey, ${ }^{5}$ Experimental Animal Center (MEDITAM), Istanbul Medipol University, Istanbul, Turkey, ${ }^{6}$ Istanbul Yeditepe University, Faculty of Pharmacy, Department of Pharmacognosy, Istanbul,Turkey, and ${ }^{7}$ Istanbul Medipol University, Regenerative and Restorative Medical Research Center (REMER), Istanbul, Turkey

"The first two authors had equal contribution.

Address for correspondence: M.Y. Gunal, MD, Assoc Prof, Alanya Alaaddin Keykubat University, School of Medicine, Department of Physiology, 07450 Antalya, Turkey.

Phone: +90 2425106135 forces in incisional wound models. Besides, histological evaluations are of great importance in these studies. The stress (Mpa) parameter measured in the axonal tensile tests used in incisional wound measurement is defined as the force value affecting at the unit area and is also called tensile stress. Strain $(\mathrm{mm} / \mathrm{mm})$ is defined as the degree of deformation according to the state of the skin sample under load before the load is applied. This definition is a mathematical concept that describes the distortion of all materials showing elastic properties. Percent elongation (\% elongation) is the change of percent difference between the length of the skin tissue before starting the tensile test and the skin length just before the skin rupture occurs (2).

Today extracts obtained from plants, monoterpenes such as carvacrol, various essential oils and many drugs, are used to regulate the wound healing process (3-5). Carpobrotus edulis (L.) N.E.Br. is an invasive plant species belonging to the family of Aizoaceae, which is unique to the Cape Region of South Africa (6-8). This plant is used by the people of this region as a traditional medicine for the treatment of a wide range of ailments such as tuberculosis, throat infections, burns, and stomach ailments (9-11). It is also used in Tunisia and its environs for its wound healing properties (12). Studies have shown that $C$. edulis extract has a perfect inhibitory effect against NO production and therefore has anti-inflammatory activity (13). In addition, because of the potent antioxidant properties of flavonoids contained in the traditional treatment method is used, justifies the choice of treatment (14, 
Tab. 1. Liposome formulation and composition.

\begin{tabular}{llccc}
\hline & Composition & $\mathrm{pH}$ & Molar Ratio & Observations \\
\hline CEPE free & *HPC:DCP:CHOL & 5.5 & $7: 1: 2$ & Milky dispersion \\
18\%CEPE & *HPC:DCP:CHOL+C.edulis (18\% w/v) & 5.5 & $7: 1: 2$ & Milky dispersion \\
\hline *HPC: Hydrogenated phosphatidyl choline; DCP: Dicetyl phosphate; CHOL: Cholesterol, CEPE: Carpobrotus \\
edulis powder extract liposomal formulation
\end{tabular}

Tab. 2. Characterization result of herbal liposome formulations.

\begin{tabular}{llccc}
\hline & Composition & $\begin{array}{c}\text { Size } \\
(\mathrm{nm})\end{array}$ & $\begin{array}{c}\text { Zeta Potential } \\
(\mathrm{mV})\end{array}$ & PDI \\
\hline CEPE free & *HPC:DCP:CHOL & $158.80 \pm 2.88$ & $-24.8 \pm 0.18$ & $0.144 \pm 0.001$ \\
$18 \%$ CEPE & *HPC:DCP:CHOL+C.edulis $(18 \% \mathrm{w} / \mathrm{v})$ & $178.80 \pm 7.42$ & $-25.4 \pm 0.62$ & $0.172 \pm 0.003$ \\
\hline *HPC: Hydrogenated phosphatidyl choline; DCP: Dicetyl phosphate; CHOL: Cholesterol; PDI: Poly Dispersity \\
Index; CEPE: Carpobrotus edulis powder extract liposomal formulation
\end{tabular}

15). It has been shown that C.edulis phytochemicals can induce apoptosis in cancer cells and is involved in the regulation of key proteins. In the same study, agar diffusion test was carried out to evaluate its antibacterial effect and as a result, the phenolic extract of C.edulis showed strong antibacterial activity against Bacillus cereus, Staphylococcus aureus and S. epidermidis (12).

Centella asiatica (L.) Urban Extract (CAE) is another traditional herbal extract commonly used for therapeutic purposes and particularly in dermatological formulations. It is particularly useful in the repair of wound tissue and has also potent antimicrobial activity. Therefore, it was preferred as a reference material in our study (16).

Skin absorption properties of molecules used topically for wound healing are factors that directly affect the healing process. Nanoencapsulation can increase the absorption of molecules in target tissues and increase the chemical stability and bioavailability of the molecules (17). The use of liposomes as a drug or molecule carrier provides sufficient penetration of these molecules to the target tissue while minimizing systemic side effects (18). No significant impact and changes were observed in the skin structure after the application of empty liposomes to the skin (19-21).

Although C. edulis is a native plant to South Africa, it is easily cultivated widespread in the coastal areas of Turkey to overlay bare lands as an invasive plant with beautiful flowers. Due to its widespread distribution people use the fleshy part of leaves for wound healing and also is applied on skin as an anti-ageing remedy. In this study, we aimed to investigate the effect of the liposomal formulation containing $C$. edulis extract on the incisional and excisional wound healing process.

\section{Materials and methods}

\section{Test material}

Succulent leaves of C. edulis (L.) N.E.Br. were collected from Yeditepe University Forest area (Istanbul) in April (40.972748 latitude and 29.152182 longitude). Fleshy parts of fresh leaves were removed $(200 \mathrm{~g})$ and homogenized in distilled water (400 $\mathrm{mL}$ ). The homogenate was then frozen $\left(\right.$ at $-80{ }^{\circ} \mathrm{C}$ ) and lyophilized (yield $2.35 \mathrm{~g}$ ).
Preparation of herbal liposome formulations containing Carpobrotus edulis powder extract (CEPE)

The chemicals used to prepare the liposome formulations were purchased from the following companies $(\mathrm{HPC}=$ Hydrogenated phosphatidylcholine, LIPOID GmbH, Germany; DCP = Dicetyl phosphate, Sigma Aldrich, USA; Cholesterol, Fluka Research Chemicals, USA).

Herbal liposome dispersion formulation was prepared by thin film methods. Firstly, liposome dispersion was prepared by dissolving the $100 \mu \mathrm{M}$ of phospholipids) in $40 \mathrm{~mL}$ organic solvent (chloroformmethanol) mixture in a round-bottom flask. The organic solvent was evaporated using a rotary evaporator under reduced pressure to form a thin film over the wall of round-bottom flask. The dried lipoid film was hydrated over a water bath with $10 \mathrm{mM}$ phosphate buffer solution containing hydrophilic active substance (CEPE) at $\mathrm{pH} 5.5$ above phase transition temperature. After multi lamellar vesicle preparation, ultrasonication process (Hiescher, UP400, Germany) and physical incorporation technique were applied, respectively. Final concentration of CEPE was $18 \%$ (w/v) (Tab. 1). Then liposomes were sonicated during 5 minutes $\mathrm{x} 3$ times to provide content uniformity. All dispersions were kept under nitrogen atmosphere at $4{ }^{\circ} \mathrm{C}$ until experimental processing.

Once the liposomes were prepared, their sizes, zeta potentials and PDI (Poly Dispersity Index) values were measured, respectively. According to the results obtained, the mean particle size distribution of sham liposomes is lower than that of CEPE loaded liposomes because of encapsulation process (Tab. 2). $18 \%$ CEPE formulation was optimized based on their PDI and zeta potential values. The zeta potential is a function of the general charge of a particle and it gives a secondary stability parameter of liposome dispersions. Differences in particle size give information about aggregation and fusion events. If the zeta potential rises above -25 $\mathrm{mV}$ and $+25 \mathrm{mV}$ (away from zero) stability increases. As this potential value approaches zero, the product loaded with liposomes breaks down quickly. In this research, $-24.8 \mathrm{mV}$ for empty liposome formulation, $-25.4 \mathrm{mV}$ for $18 \% \mathrm{CEPE}$ were found for optimum liposome formulations with zeta potential. Therefore, it is seen that the liposomes we have used have a stable structure. The value of PDI is an indication of the rate of degradation of liposomes. As the PDI value increases, the rate of degradation of the liposome increases (22). When liposomes were evaluated from the point of view of PDI, CEPE-loaded liposomes had a larger particle size than empty liposomes, and PDI values were between 0.1 and 0.5 , which is the ideal value. According to all these information, liposomal dispersions containing 18\% CEPE preferred because they have an encapsulation property and appropriate zeta potentials.

\section{Animals}

Male Wistar-Albino rats weighing 250-300 gr were used in the study. The rats were hosted in regular cages with food and water 
912-917

A

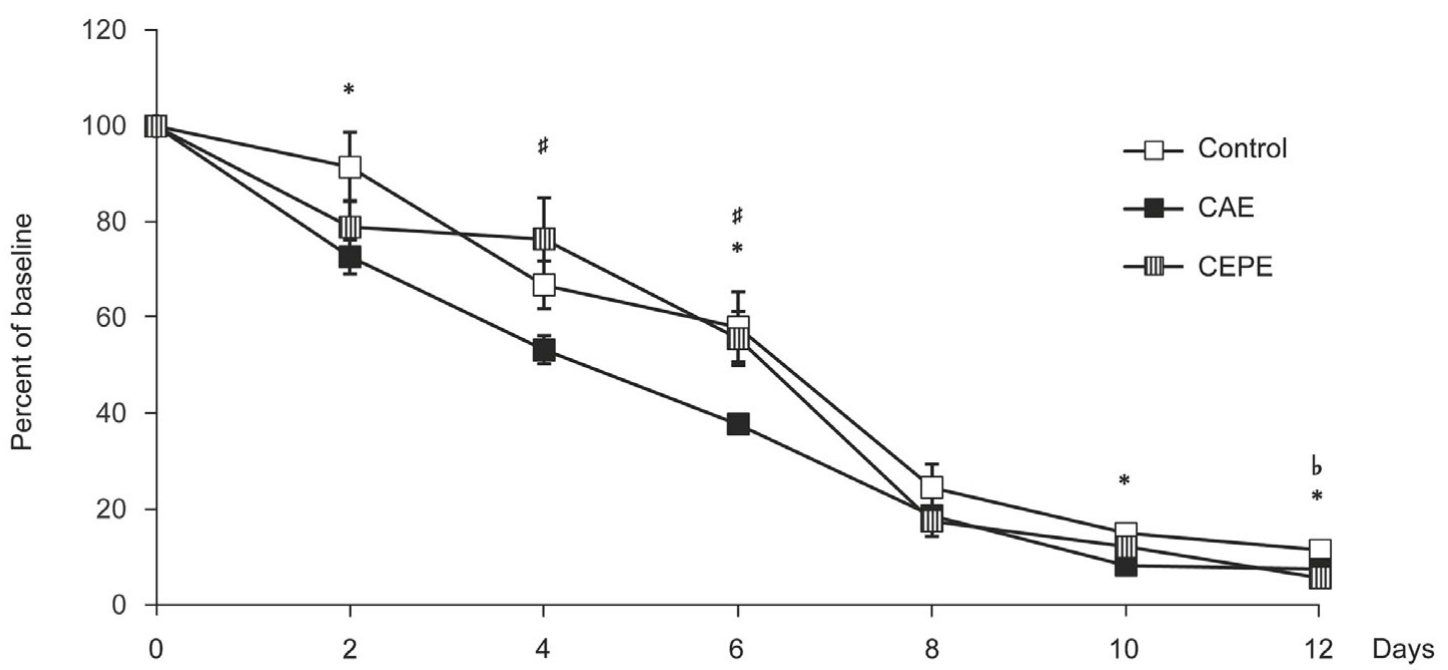

(B)

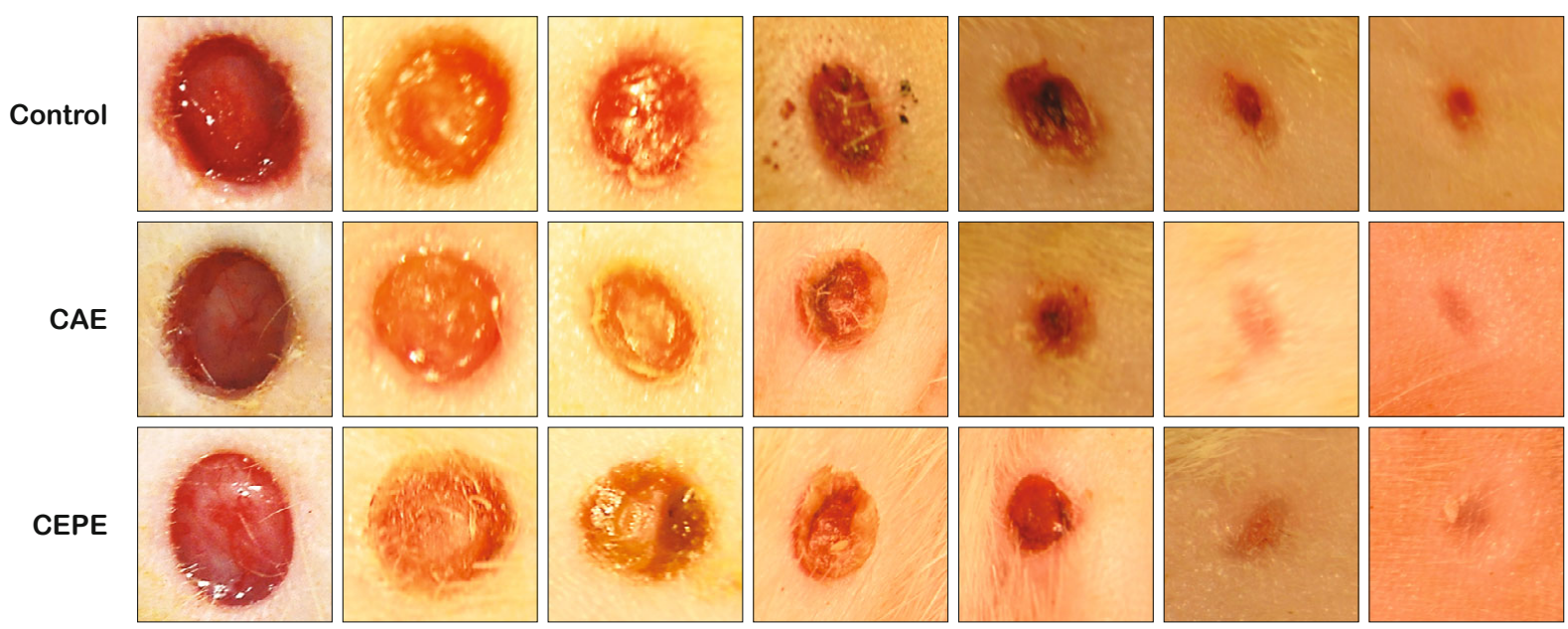

Fig. 1. The effect of C.edulis powder extract liposomal formulation (CEPE) on the wound healing process in the excisional wound model. A. Healing percentage of wound tissue surface area in each group. * $\mathbf{p}<0.05$ control group compared with $\mathrm{CAE}$ group; ${ }^{\mathrm{b}} \mathbf{p}<0.05$ control group compared with CEPE group; $\sharp p<0.05$ CAE group compared with CEPE group. All values are given as mean \pm S.E.M. B. Macroscopic photographs of wound tissue.

ad libitum, at room temperature $\left(24^{\circ} \mathrm{C}\right)$ with artificial light from 7.00 am to $7.00 \mathrm{pm}$. Before performing in vivo experiments, ethical clearance approval was obtained from the Local Ethical Committee. The experimental animals were divided into 3 groups of 8 animals per group; namely control group, the group treated commercial product containing $1 \%$ Centella asiatica extract (CAE) as reference drug and the group treated test material containing $18 \%$ Carpobrotus edulis powder extract liposomal formulation (CEPE).

\section{Anesthesia and protocols for wound induction}

Rats were anesthetized with a combination of $60 \mathrm{mg} / \mathrm{kg}$ ketamine and $6 \mathrm{mg} / \mathrm{kg}$ xylazine intraperitoneally (4). The modified wound model was used to create the wound model considering the information in the literature $(4,23)$. Two excisional wound tissues were formed using a $5 \mathrm{~mm}$ punch biopsy instrument on the left side of the midline in the shaved back region, one $\mathrm{cm}$ apart from each other and $1.5 \mathrm{~cm}$ away from the midline. Except for the control group, linear full-thickness incisional wound was formed in a length of $2 \mathrm{~cm}$ using a No.11 surgical scalpel to remain $1.5 \mathrm{~cm}$ to the right of the midline in the dorsal region. Incisional wound edges were sutured using $3 / 0$ silk thread. In the control group, only the excisional wound model was formed as described above. Drug formulations were administered topically to the relevant groups every morning for 11 days. On day 12 of the study, the rats were sacrificed under anesthesia; tissue samples were taken for histological evaluation and wound tension measurement.

\section{Macroscopic evaluation}

From the day when the excisional wound tissue was formed, every other day photographs were taken, and the surface areas were measured with the ImageJ program. Afterwards, wound healing rates were calculated by the formula given in the literature $(4,5)$. 

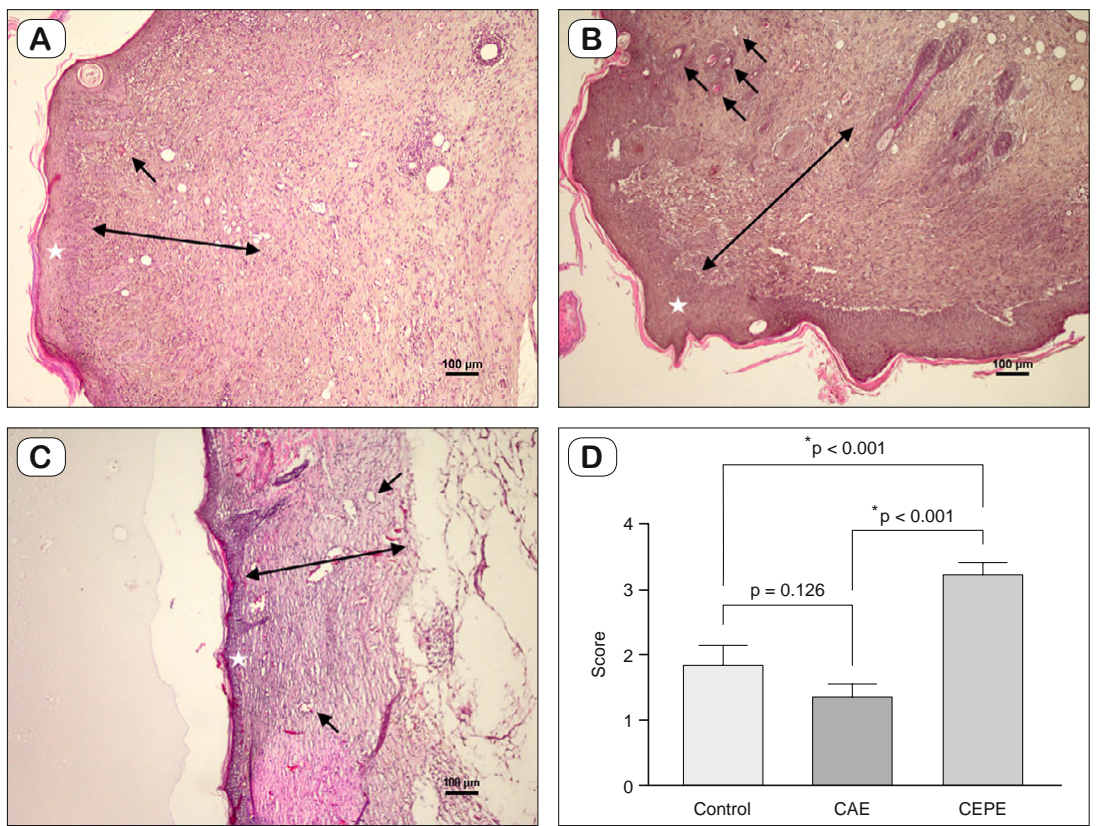

Fig. 2. Histological evaluation of wound tissue (Hemotoxylin and eosin (H\&E), (original magnification $x$ 10)). The scale bars represent $100 \mu \mathrm{m}$ for a figure. A. Control group, B. CAE group, C. CEPE group, D. Histological scoring of wound tissues. In the histological scoring of the healing of the excisional wound tissue; epidermal regeneration (it is illustrated by " $\star$ " in the figure), dermal regeneration and granulation tissue (it is illustrated by " $\leftrightarrow$ " in the figure), and angiogenesis (it is illustrated by $" \rightarrow$ " in the figure) were better in the CEPE group than in the control group.

\section{Histological evaluation}

On day 12 , the animals were sacrificed by decapitation and the skin of the back including the wound area was removed. Fullthickness biopsy samples extended from the outside margin to the center of the treated area. All tissue specimens were fixed in $10 \%$ neutral formalin for at least $24 \mathrm{~h}$ at room temperature. After fixation, wound samples were dehydrated in graded ethanol series, cleared in xylene and embedded in paraffin. $5 \mu \mathrm{m}$ thick sections (Thermo Microm HM 340E, Waltham, US) were mounted on glass slides, dewaxed, rehydrated with distilled water and stained with Hematoxylin-Eosin (HE) according to routine procedures for light microscopy. The images were taken under a Nikon Eclipse $\mathrm{Ni}$ (Nikon Instruments Europe BV, Amsterdam, Holland) research microscope. Measurement was performed by two independent researchers blind to the drug administration groups. The scoring system defined by Galeano et al. was used for the histological evaluation of wound healing (24-26).

Axial tensile-elongation experiments in incisional wound tissue

Axial tensile-elongation experiments were carried out on a TA Instruments QA-800 mechanical analyzer at Yeditepe University Engineering Faculty. In order to standardize the data obtained from the skin samples taken after the incisional wound model, the crosssectional areas of skin tissues to be subjected to tensile-elongation tests were measured separately. During the measurement, the skin tissues were attached to the fixed ends of the mechanical analyzer.
All pull tests were initiated by resetting the force sensor. At certain intervals, a draw of 100 microns was applied. At the same time, the device continued to record by continuously measuring the stress-strain responses with applied force. Maximum load, maximum stress, strain and percent elongation values calculated in skin tissue.

\section{Statistical evaluation}

For statistical data comparisons, a standard software package (SPSS 20 for Windows; SPSS Inc., Chicago, IL, USA) was used. Differences between groups were analyzed by one-way ANOVA, followed by least significant differences (LSD) tests. All values were given as mean \pm S.E.M. $p<0.05$ were considered significant.

\section{Results}

There was no significant difference between the groups when compared with the control group at 8 th day in the evaluation of the surface of the excisional wound. However, there was a significant difference on the 2nd, 6th, 10th and 12th days, between $\mathrm{CAE}$ and the control group (respectively $\mathrm{p}=0.029 ; \mathrm{p}=0.016 ; \mathrm{p}=0.01 ; \mathrm{p}=0.03) ;$ on the 4th and 6th days, between the CAE group and CEPE group (respectively $\mathrm{p}=0.014 ; \mathrm{p}=0.007)$; on the 12 th day between the control group and CEPE groups $(p=0.004)$ (Fig. 1).

Histological scoring of excisional wound tissue was evaluated for epidermal regeneration, dermal regeneration, and granulation tissue formation and angiogenesis. The values of the CEPE group were higher than both, the control group and the CAE group ( $\mathrm{p}<$ $0.001 ; \mathrm{p}<0.001$, respectively) (Fig. 2).

When the maximum stress, strain, and elongation levels of the incisional wound tissue were evaluated, there was a statistically significant decrease in all parameters in the CAE and CEPE groups compared to the control group. This situation was expected because the skin tissue of control group was intact. There was no significant difference between CAE and CEPE (Fig. 3).

\section{Discussion}

Wound healing is a complex process consisting of inflammation, cellular proliferation and remodeling (1). Many molecules can accelerate wound healing by affecting these phases. A wide range of herbal remedies either directly or its extracts such as ethanol or oily extracts of Hypericum perforatum (27) and various essential oils such as Lavender oil (28) were also used for this purpose in worldwide communities. Although C. edulis is native to South Africa's Cape region, it is an invasive plant species that are common throughout the world (6-8). Traditionally, it is widely used 
(A)

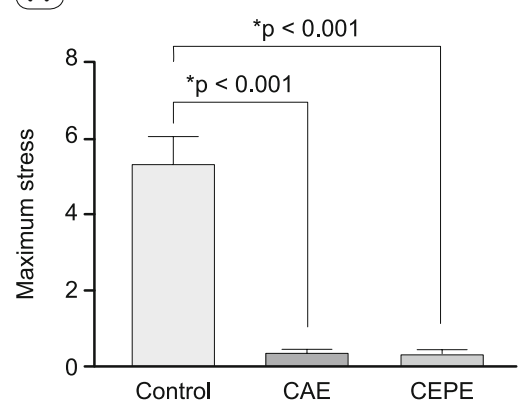

(B)

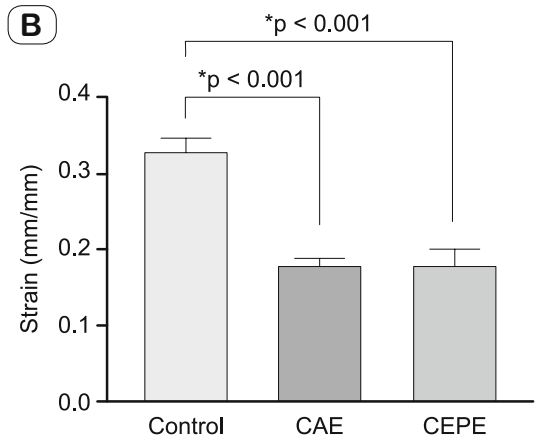

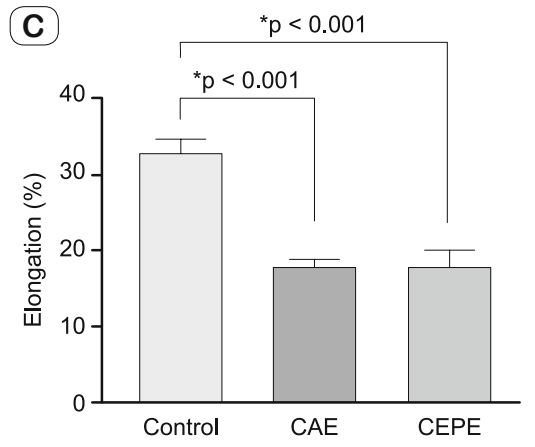

\begin{tabular}{lcrr|}
\hline & CONTROL & \multicolumn{1}{c}{ CAE } & \multicolumn{1}{c}{ CEPE } \\
\hline Maximum stress (Mpa) & $5,37778 \pm 0,68375$ & $0,37843 \pm 0,04259$ & $0,32867 \pm 0,07325$ \\
\hline Strain (mm/mm) & $0,32925 \pm 0,01616$ & $0,17983 \pm 0,00736$ & $0,17865 \pm 0,01901$ \\
\hline Elongation (\%) & $32,86508 \pm 1,2605$ & $17,95805 \pm 0,73563$ & $19,03871 \pm 1,60914$ \\
\hline
\end{tabular}

*All values are given as mean \pm S.E.M.

Fig. 3. Axial tensile-elongation measurements in incisional wound tissue. A. Maximum stress values, B. Strain values, C. Elongation (\%) values. All values are given as mean \pm S.E.M.

by local people in the treatment of respiratory infections, various stomach ailments, wounds, and burns (9-12).

Wound healing studies are generally performed with the burn, incisional, and excisional wound models. In our research, incisional and excisional wound models were used together, as stated in the material-method section to use fewer experimental animals. The photographs of the excisional wounds were evaluated with the Image J program and surface area healing rates were calculated. According to the measurement results, the recovery rates of CAE and CEPE groups were significantly better, especially after the eighth day (Fig. 1).

Angiogenesis, granulation tissue formation, dermal, and epidermal regeneration are the main histological evaluation criteria for wound healing. In our study, biopsy materials were taken from excisional wound tissues and evaluated as described in the literature $(24,25)$. In histological evaluation, angiogenesis, epidermal regeneration, size and organization of granulation tissue and dermal regeneration were better in the CEPE group compared to both the control group and the CAE group (Fig. 2).

In the evaluation of incisional wound tissue, it is important to measure the wound tissue tension forces. Fibroblast cell proliferation is the leading factor affecting wound tension. These cells convert to myofibroblast form and contribute to wound tension. Axial tensile elongation tests were applied to the samples taken from the incisional wound tissues to evaluate the wound tensile strength. When the control group and other groups were compared, there was a statistically significant difference in terms of maximum stress, strain, and percent elongation values (Fig. 3). These parameters in the control group were better due to undamaged skin tissue. On the other hand, CAE and CEPE groups were not statistically significant in their comparison, and the values were similar. Also, the histological evaluation parameters in our study were consistent with the data obtained in the incisional axial-tensile elongation test.

Infectious agents, such as bacteria, can adversely affect the wound healing process, slowing or completely disrupting the heal- ing process. Therefore, the antibacterial properties of the molecules to be used in the treatment affect the healing positively and are preferred. It has been found that a large number of compounds obtained from C.edulis show antibacterial properties (11). It has been shown to have vigorous antibacterial activity, especially against triterpene uvaol, multidrug-resistant (MDR) Gram-positive bacteria (29). Interestingly, in another study with methanol extract of the same plant, it was suggested that this extract could be used against problematic MDR intracellular infections (30).

In light of all these information, C.edulis extract may be a new option in wound healing because of the similar properties of CAE, which is accepted as the reference molecule in routine use in wound tissues for treatment purposes, and also it has strong antibacterial properties.

Liposomal formulations of C.edulis extract have positive effects on the healing process in both incisional and excisional wound tissues. Our research is a preliminary study, and we believe that further investigations on its crude extract and fractions as well as its purified components by using molecular methods would provide data to shed light on new treatment modalities.

\section{Limitations}

Although the effect of liposomal formulations of CAE and C.edulis extracts on wound healing was investigated, parameters such as cytokine and growth factors were not evaluated.

\section{References}

1. Singer AJ, Clark RA. Cutaneous wound healing. N Engl J Med 1999; 341 (10): 738-746.

2. Remache D, Caliez M, Gratton M, Dos Santos S. The effects of cyclic tensile and stress-relaxation tests on porcine skin. J Mech Behav Biomed Mater 2018; 77: 242-249. 
3. Ayla S, Okur ME, Gunal MY et al. Wound healing effects of methanol extract of Laurocerasus officinalis roem. Biotechnic Histochemistry 2019; 94 (3): 180-188.

4. Gunal MY, Heper AO, Zaloglu N. The Effects of Topical Carvacrol Application on Wound Healing Process in Male Rats. Phcog J 2014; 6 (3): 10-14.

5. Okur ME, Ayla S, Polat DC, Gunal MY, Yoltas A, Biceroglu O. Novel insight into wound healing properties of methanol extract of Capparis ovata Desf. var. palaestina Zohary fruits. J Pharm Pharmacol 2018; 70 (10): 1401-1413.

6. Conser C, Connor EF. Assessing the residual effects of Carpobrotus edulis invasion, implications for restoration. Biol Invasions 2009; 11 (2): 349-358.

7. Fenollosa E, Roach DA, Munne-Boscht S. Death and Plasticity in Clones Influence Invasion Success. Trends Plant Sci 2016; 21 (7): 551-553.

8. Roiloa SR, Rodriguez-Echeverria S, Freitas H, Retuerto R. Developmentally-programmed division of labour in the clonal invader Carpobrotus edulis. Biol Invasions 2013; 15 (9): 1895-1905.

9. Mathabe MC, Nikolova RV, Lall N, Nyazema NZ. Antibacterial activities of medicinal plants used for the treatment of diarrhoea in Limpopo Province, South Africa. J Ethnopharmacol 2006; 105 (1-2): 286-293.

10. Thring TSA, Weitz FM. Medicinal plant use in the Bredasdorp/Elim region of the Southern Overberg in the western cape province of South Africa. J Ethnopharmacol 2006; 103 (2): 261-275.

11. van der Watt E, Pretorius JC. Purification and identification of active antibacterial components in Carpobrotus edulis L. J Ethnopharmacol 2001; 76 (1): 87-91.

12. Meddeb E, Charni M, Ghazouani T et al. Biochemical and Molecular Study of Carpobrotus edulis Bioactive Properties and Their Effects on Dugesia sicula (Turbellaria, Tricladida) Regeneration. Appl Biochem Biotechnol 2017; 182 (3): 1131-1143.

13. Ondua M, Njoya EM, Abdalla MA, McGaw LJ. Anti-inflammatory and antioxidant properties of leaf extracts of eleven South African medicinal plants used traditionally to treat inflammation. J Ethnopharmacol 2019; 234: 27-35.

14. Hafsa J, Hammi KM, Khedher MRB et al. Inhibition of protein glycation, antioxidant and antiproliferative activities of Carpobrotus edulis extracts. Biomed Pharmacother 2016; 84: 1496-503.

15. Omoruyi BE, Bradley G, Afolayan AJ. Antioxidant and phytochemical properties of Carpobrotus edulis (L.) bolus leaf used for the management of common infections in HIV/AIDS patients in Eastern Cape Province. BMC Complement Altern Med 2012; 12: 215.

16. Yao CH, Yeh JY, Chen YS, Li MH, Huang CH. Wound-healing effect of electrospun gelatin nanofibres containing Centella asiatica extract in a rat model. J Tissue Eng Regen Med 2017; 11 (3): 905-915.
17. Bonechi C, Martini S, Ciani L et al. Using Liposomes as Carriers for Polyphenolic Compounds: The Case of Trans-Resveratrol. Plos One 2012; 7 (8).

18. Carita AC, Eloy JO, Chorilli M, Lee RJ, Leonardi GR. Recent Advances and Perspectives in Liposomes for Cutaneous Drug Delivery. Curr Med Chem 2018; 25 (5): 606-635.

19. Duman G, Aslan I, Ozer AY, Inanc I, Taralp A. Liposome, gel and lipogelosome formulations containing sodium hyaluronate. J Liposome Res 2014; 24 (4): 259-269.

20. Pierre EJ, Perez-Polo JR, Mitchell AT, Matin S, Foyt HL, Herndon DN. Insulin-like growth factor-I liposomal gene transfer and systemic growth hormone stimulate wound healing. J Burn Care Rehabil 1997; 18 (4): 287-291.

21. Spies M, Nesic O, Barrow RE, Perez-Polo JR, Herndon DN. Liposomal IGF-1 gene transfer modulates pro- and anti-inflammatory cytokine mRNA expression in the burn wound. Gene Ther 2001; 8 (18): 1409-1415.

22. Pham TT, Jaafar-Maalej C, Charcosset C, Fessi H. Liposome and niosome preparation using a membrane contactor for scale-up. Colloid Surface B 2012; 94: 15-21.

23. Reid RR, Said HK, Mogford JE, Mustoe TA. The future of wound healing: pursuing surgical models in transgenic and knockout mice. J Am Coll Surg 2004; 199 (4): 578-585.

24. Altavilla D, Saitta A, Cucinotta D et al. Inhibition of lipid peroxidation restores impaired vascular endothelial growth factor expression and stimulates wound healing and angiogenesis in the genetically diabetic mouse. Diabetes 2001; 50 (3): 667-674.

25. Galeano M, Altavilla D, Cucinotta $D$ et al. Recombinant human erythropoietin stimulates angiogenesis and wound healing in the genetically diabetic mouse. Diabetes 2004; 53 (9): 2509-17.

26. Niwano Y, Koga H, Sakai A et al. Wound healing effect of malotilate in rats. Arzneimittelforschung 1996; 46 (4): 450-5.

27. Suntar IP, Akkol EK, Yilmazer D et al. Investigations on the in vivo wound healing potential of Hypericum perforatum L. J Ethnopharmacol 2010; 127 (2): 468-477.

28. Mori HM, Kawanami H, Kawahata H, Aoki M. Wound healing potential of lavender oil by acceleration of granulation and wound contraction through induction of TGF-beta in a rat model. Bmc Complem Altern M 2016; 16.

29. Martins A, Vasas A, Viveiros M, Molnar J, Hohmann J, Amaral L. Antibacterial properties of compounds isolated from Carpobrotus edulis. Int J Antimicrob Agents 2011; 37 (5): 438-444.

30. Martins M, Ordway D, Kristiansen M et al. Inhibition of the Carpobrotus edulis methanol extract on the growth of phagocytosed multidrugresistant Mycobacterium tuberculosis and methicillin-resistant Staphylococcus aureus. Fitoterapia 2005; 76 (1): 96-99. 